\title{
Neutralizing Antibodies against SARS-CoV-2 and HLA Class I and II Polymorphism
}

\author{
Lisa Weidner $^{a} \quad$ Julia Kalser ${ }^{a} \quad$ Thomas R. Kreil $^{b} \quad$ Christof Jungbauer $^{a}$ \\ Wolfgang R. Mayr ${ }^{a}$ \\ ${ }^{a}$ Austrian Red Cross, Blood service for Vienna, Lower Austria and Burgenland, Vienna, Austria; \\ ${ }^{b}$ Global Pathogen Safety, Baxter AG, a Takeda company, Vienna, Austria
}

\section{Dear Editor,}

In some infectious diseases, the humoral immune response against pathogens is associated with HLA class I and/or class II alleles (see e.g. [1]). Novelli et al. [2] described a correlation between some polymorphisms and severe COVID-19 disease. Therefore, it was interesting to analyze whether or not an association exists between the production of neutralizing antibodies against SARSCoV-2 and HLA class I and/or class II polymorphisms.

Therefore, 84 individuals were randomly selected out of the 100 plasma donors being convalescent after SARSCoV-2 infection (characterization of SARS-CoV-2 antibodies previously published [3]) and analyzed for HLA class I and class II polymorphisms. HLA genotyping for HLA-A, -B, -C, -DRB1, -DQB1, and -DPB1 alleles was performed by massive parallel sequencing using NGSgo ${ }^{\circledR}$ (GenDx, Utrecht, The Netherlands) with DNA isolated from blood and analyzed on Miseq platform (Illumina, San Diego, USA) in 84 plasma donors and in 100 healthy individuals of the same population (Caucasoids living in Vienna, Austria). The statistical evaluations have been done by using the $\chi^{2}$ test with Yates' correction for continuity in $2 \times 2$ contingency tables where the antibody status was compared with the presence or the absence of the single HLA alleles. Due the enormous HLA polymorphism, only data of low resolution genotyping have been analyzed (HLA-A: 20, HLA-B: 35, HLA-C: 14, HLA-DRB1: 13, HLA-DQB1: 5, and HLA-DPB1: 23 specificities).

\section{karger@karger.com} www.karger.com/tmh

Karger"

BOPEN ACCESS
(C) 2021 The Author(s)

Published by S. Karger AG, Basel

This is an Open Access article licensed under the Creative Common Attribution-NonCommercial-4.0 International License (CC BY-NC) (http://www.karger.com/Services/OpenAccessLicense), applicable to the online version of the article only. Usage and distribution for commercial purposes requires written permission.
There was no significant difference in the allele frequencies of the plasma donors and of the controls, thus showing that both samples originate from the same population and indicating that the production of SARS-CoV2 antibodies is not strongly influenced by HLA polymorphisms.

The quantity of neutralizing antibodies against SARSCoV-2 in the plasma donors tested between 26 and 61 days after onset of symptoms followed a normal distribution (range: $1:<7.7$ to $1: 1,765.0$, mean: $1: 231$ ). The majority (98.8\%) of donors included had COVID-19 symptoms that classify as WHO $\leq 4$.

Two analyses on antibody carriers have been performed.

- comparing the HLA polymorphisms of the low and high titer donors (titers lower than mean minus 1 standard deviation versus titers higher than mean plus one standard deviation, each group $n=14$ ), and

- dividing the titers into 3 groups of $1 / 3$ and comparing the HLA polymorphisms of the low and the high titer groups.

Both comparisons showed no differences in the frequency of HLA class I and/or class II alleles in the respective groups.

The 3 most frequent alleles in the 6 loci were in the control population: HLA-A ${ }^{*} 02,{ }^{*} 03$, and ${ }^{*} 01$; HLA$\mathrm{B}^{*} 07, * 12$, and $* 08$; HLA-C*07, $* 03$, and $* 02$; HLADRB $1 * 01, * 15$, and $* 07$; HLA-DQB $1^{*} 03, * 02$, and ${ }^{*} 06$; and DPB $1^{*} 04, * 02$, and ${ }^{*} 03$. These alleles represent also 
the most frequent alleles in the groups of high or low titered donations of the second comparison with one exception: donors with low titered donations showed a higher allele frequency of HLA-B15 (0.16 vs. 0.09 in controls). This difference, however, is not significant at the level of $p=0.05$ due to the relatively low number of individuals tested.

In conclusion, it can be deduced that in a Caucasoid population of Central Europe, the HLA class I and/or class II polymorphisms analyzed do not seem to play a major role in the quantity of neutralizing antibodies produced against SARS-CoV-2. Due to the relatively low number of individuals tested and the prodigious polymorphism of the HLA system, the statistical evaluation has a limited power. For this reason, it is recommended to corroborate with a larger sample of carriers of antibodies after SARS-CoV-2 infection.

\section{Statement of Ethics}

In this study, human residual serum for routine laboratory diagnostics as conducted in the course of convalescent plasma donation workup according to European and local legal regulations was used. All donors of the samples used signed informed consent on the use of leftover material for research purposes. The work described has been carried out in accordance with The Code of Ethics of the World Medical Association (Declaration 129 of Helsinki). Samples were processed anonymously to protect privacy of each donor.

\section{Conflict of Interest Statement}

T.R.K. has Takeda stock interest. All other authors declare no conflict of interests.

\section{Funding Sources}

This research did not receive any specific grant from funding agencies in the public, commercial, or not-for-profit sectors.

\section{Author Contributions}

W.R.M. designed the study and interpreted the data, J.K. performed HLA typing, T.R.K. provided data of the SARS-CoV-2 neutralization assay, and C.J. and L.W. provided donor information and critically reviewed the manuscript. All authors critically revised and approved the manuscript.

\section{References}

1 Scepanovic P, Alanio C, Hammer C, Hodel F, Bergstedt J, Patin E, et al.; Milieu Intérieur Consortium. Human genetic variants and age are the strongest predictors of humoral immune responses to common pathogens and vaccines. Genome Med. 2018 Jul;10(1):59.

2 Novelli A, Andreani M, Biancolella M, Liberatoscioli L, Passarelli C, Colona VL, et al. HLA allele frequencies and susceptibility to COVID-19 in a group of 99 Italian patients. HLA. 2020 Nov;96(5):610-4.

3 Weidner L, Gänsdorfer S, Unterweger S, Weseslindtner L, Drexler C, Farcet M, et al. Quantification of SARS-CoV-2 antibodies with eight commercially available immunoassays. J Clin Virol. 2020 Aug;129(July):104540. 